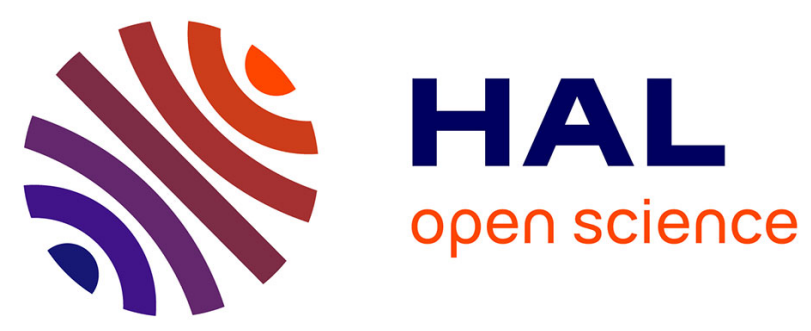

\title{
Légitimation et disqualification par l'histoire dans les manifestations de rue en Russie (2011-2016)
}

Alexandra Arkhipova, Dmitry Doronin, Elena Iougaï, Anna Kirziouk, Darya Radtchenko, Alexeï Titkov, Anna Volkova, Benjamin Guichard

\section{To cite this version:}

Alexandra Arkhipova, Dmitry Doronin, Elena Iougaï, Anna Kirziouk, Darya Radtchenko, et al.. Légitimation et disqualification par l'histoire dans les manifestations de rue en Russie (2011-2016). Le Mouvement social, 2017, 260 (3), pp.129-148. 10.3917/lms.260.0129 . halshs-02422312

\section{HAL Id: halshs-02422312 \\ https://shs.hal.science/halshs-02422312}

Submitted on 14 Mar 2020

HAL is a multi-disciplinary open access archive for the deposit and dissemination of scientific research documents, whether they are published or not. The documents may come from teaching and research institutions in France or abroad, or from public or private research centers.
L'archive ouverte pluridisciplinaire HAL, est destinée au dépôt et à la diffusion de documents scientifiques de niveau recherche, publiés ou non, émanant des établissements d'enseignement et de recherche français ou étrangers, des laboratoires publics ou privés. 


\title{
LÉGITIMATION ET DISQUALIFICATION PAR L'HISTOIRE DANS LES MANIFESTATIONS DE RUE EN RUSSIE (2011-2016)
}

\author{
Alexandra Arkhipova, Dmitry Doronin, Elena Iougaï, Anna Kirziouk, Darya \\ Radtchenko, Alexeï Titkov, Anna Volkova, article traduit du russe par Benjamin \\ Guichard
}

La Découverte | « Le Mouvement Social »

2017/3 n² 260 | pages 129 à 148

ISSN 0027-2671

ISBN 9782707197504

Article disponible en ligne à l'adresse :

https://www.cairn.info/revue-le-mouvement-social-2017-3-page-129.htm

Distribution électronique Cairn.info pour La Découverte.

(C) La Découverte. Tous droits réservés pour tous pays.

La reproduction ou représentation de cet article, notamment par photocopie, n'est autorisée que dans les limites des conditions générales d'utilisation du site ou, le cas échéant, des conditions générales de la licence souscrite par votre établissement. Toute autre reproduction ou représentation, en tout ou partie, sous quelque forme et de quelque manière que ce soit, est interdite sauf accord préalable et écrit de l'éditeur, en dehors des cas prévus par la législation en vigueur en France. Il est précisé que son stockage dans une base de données est également interdit. 


\title{
Légitimation et disqualification par l'histoire dans les manifestations de rue en Russie (2011-2016)
}

\author{
par Alexandra ArKhipova, Dmitry Doronin, Elena Iougaï, \\ Anna Kirziouk, Daria Radtchenko, Alexeï Titkov et Maria Volkova*
}

E n septembre 2014, alors que notre groupe de recherche observait la « Marche de la paix ", organisée à Moscou et à Saint-Pétersbourg pour protester contre la guerre en Ukraine, nous avons été surpris par l'apparition de slogans jusqu'alors inconnus dans les manifestations de rue, comme "J'ai honte pour 1939, 1968 et 2014 ». Depuis, de très nombreuses références historiques ont été relevées dans les slogans de presque tous les meetings politiques organisés dans les grandes villes de Russie. Ce recours au passé pour décrire la situation présente est un phénomène nouveau qui mérite analyse et interprétation - c'est l'objet de cet article.

Du début des années 2000 à 2011, période dite de la "stabilité poutinienne ", les mouvements de protestation économique et sociale n'ont guère attiré l'attention de l'opinion publique ni des grands médias. Ceux-ci étaient considérés par la plus grande partie de la population comme des mouvements de niche issus de groupes marginaux ou d'ancrage local (minorités nationales, groupuscules d'activistes politiques, mouvements de mécontentement de retraités ou mouvements de revendications économiques à Vladivostok en 2008 et à Kaliningrad en 2009-2010). Une exception notable fut toutefois celle des émeutes et manifestations qui se déroulèrent autour de la place du Manège à Moscou en décembre 2010, en réaction au meurtre d'un supporter de football par un groupe de personnes originaires du Nord-Caucase.

Durant l'hiver 2011-2012, le résultat des élections législatives suscita un mouvement de protestation civique important, inattendu pour beaucoup, qui prit la forme au début de l'année 2012 d'une contestation ouverte de la réélection de Vladimir Poutine à la présidence de la Fédération de Russie. Ce moment est considéré comme un tournant dans l'histoire contemporaine des mouvements sociaux en Russie ${ }^{1}$, non seulement en raison de son caractère de masse, mais aussi parce qu'à partir de ce moment-là, la participation à des manifestations de rue a cessé d'être une forme d'action marginale.

En réponse à cette vague de protestations, une série de lois répressives furent adoptées, qui ont rendu plus difficile l'organisation d'actions politiques publiques et aggravé les peines encourues en cas d'infraction. De 2012 à 2016, les sanctions

* Cette recherche a été réalisée dans le cadre du projet "Mythologie et comportements ritualisés dans les villes de la Russie contemporaine ", soutenu par la Fondation scientifique de Russie [Rossijskij naučnyj fond] sous le numéro 16-18-00068 et hébergé par l'École supérieure de sciences économiques et sociales de Moscou [Moskovskaâ vysšấ škola èkonomičeskih i social'nyh nauk, MVŠÈN]. Les auteurs sont membres du groupe de recherche "Analyse du folklore contemporain " [Monitoring aktual'nogo fol'lkora, MAF]. Article traduit du russe par Benjamin Guichard.

1. A. Arhipova et M. Alekseevskij (dir.), "My ne nemy!": Antropologija protesta v Rossii 2011-2012 godov ["Nous ne sommes pas muets!" Anthropologie de la protestation sociale en Russie en 20112012], Tartu, Estonian Literature Museum, 2014. 
administratives prononcées pour participation à des actions de rue non autorisées ont été alourdies et appliquées de façon plus systématique. Cela n’a toutefois pas suffi à freiner le phénomène des manifestations. En 2012-2013, la conflictualité sociale a diminué en comparaison avec l'hiver précédent. Toutefois, les mouvements de protestation sociale ont continué et sont restés sensiblement plus nombreux qu'avant 2011. Depuis le début de l'année 2012, l'introduction de réformes des systèmes de santé et d'éducation, impliquant la diminution du nombre de postes de fonctionnaires, puis l'affaire Dima Iakovlev ${ }^{2}$, entraînant une restriction des règles d'adoption internationale, ont suscité une vague importante de contestation sociale.

Au début de l'année 2014, l'annexion de la Crimée et le conflit entre la Russie et l'Ukraine ont provoqué une nouvelle vague de manifestations de rue et d'actions publiques venues de tous les bords politiques. Les partisans de l'opposition réclamaient la fin du conflit et le respect du droit international, tandis que les partisans du gouvernement soutenaient le rattachement de la Crimée et son intégration pleine et entière à la Fédération de Russie, comme un "retour au pays » qui réparait une injustice de l'histoire. À ce moment-là, la cote de popularité du président Poutine a atteint des scores sans précédent : les études d'opinion du centre Levada ont estimé le taux de satisfaction caractérisant son action à $88 \%$ en octobre $2014^{3}$. Au printemps 2014, les États-Unis d'Amérique et l'Union européenne ont appliqué des sanctions économiques à la Russie, suscitant en réaction un embargo russe sur l'importation de certains produits européens, situation qui, à son tour, a nourri l'activisme social et politique. Une nouvelle vague de manifestations de rue a été suscitée par l'assassinat le 27 février 2015 de Boris Nemtsov, figure de l'opposition politique devenue une icône de la contestation d'inspiration libérale. Depuis le début de l'année 2016, les manifestations de chauffeurs routiers, opposés à la mise en place de nouvelles taxes sur les transports, dominent le mouvement social.

La forme des différentes actions de rue a sensiblement évolué de 2011 à 2016. Elles ne sont plus seulement une forme d'expression politique, mais participent désormais à la construction de réseaux de sociabilité et prennent même la valeur d'un loisir. La manifestation de rue est ainsi devenue une sorte de phénomène culturel urbain ; des magazines de mode et de loisirs comme Aficha et Bolchö̈ gorod y consacrent des dossiers et la question de savoir "comment s'habiller pour un meeting " est devenu un sujet journalistique ${ }^{4}$.

\section{Une typologie politique des manifestations de rue}

Les manifestations étudiées dans le cadre de cette recherche peuvent être réparties en quatre profils types, établis en fonction de leur orientation politique dominante.

Le premier profil est celui des manifestations loyalistes. Nous désignons ainsi les actions de soutien au gouvernement ainsi que l'activisme de groupes comme le

2. Du nom d'un orphelin russe décédé à la suite de mauvais traitements aux États-Unis en 2008, dont les parents adoptifs furent acquittés par la justice américaine, entraînant un vaste mouvement d'opinion xénophobe en Russie et une initiative de la Douma pour prendre des sanctions à l'égard des États-Unis, notamment la suspension des protocoles d'adoption internationale entre les deux pays. [NdT]

3. Taux d'appréciations favorables de l'action de Vladimir Poutine, indicateur publié sur le site internet du Yuri Levada Analytical Center : https:/www.levada.ru/indikatory/odobrenie-organov-vlasti.

4. "Kak odet'sâ na miting ? 7 sovetov ot Romena Kromenskgo ", chronique du journaliste Romen Kromenskij sur la chaîne télévisée Dožd’, 20 janvier 2016, disponible sur la page de la chaîne sur la plateforme Youtube : https://www.youtube.com/watch?v=-5u0IogsSto. 
mouvement "Anti-Maïdan » qui s'est développé au début de l'année 2015. Ce dernier s'était donné comme objectif de constituer une opposition "patriotique " aux mouvements de protestation antirusses des pays proches (mouvement Maïdan en Ukraine et " révolutions de couleur » dans les pays issus de l'URSS), dénoncés comme des formes d'instrumentalisation par les États-Unis d'Amérique et l'OTAN. De tels mots d'ordre sont partagés par le Mouvement de libération populaire (Narodnoosvoboditel'nö̈e dvijenie, NOD), constitué en 2012 mais qui n'a pris massivement part à des actions de rue qu'à compter du conflit ukrainien de 2014. Le NOD se caractérise par un soutien inconditionnel à la politique défendue par Vladimir Poutine, à commencer par le rattachement de la Crimée et l'aide apportée aux régions sécessionnistes d'Ukraine se réclamant de la "Nouvelle Russie ", et par une rhétorique vigoureusement hostile à l'Occident. Les partisans du NOD expriment leurs slogans dans l'espace public dans le cadre de défilés ou de piquets revendicatifs de quelques personnes. Cette dernière forme d'action leur est spécifique, ils l'utilisent notamment comme forme de contre-manifestation lors des actions de l'opposition. Les concerts-meetings en plein air, enfin, constituent une autre forme de manifestation du soutien loyaliste au pouvoir. En 2014-2016, des événements de ce type ont été organisés dans différentes régions en soutien au rattachement de la Crimée avec l'aide financière et matérielle des administrations publiques, par ailleurs tenues d'y envoyer des cohortes de participants. Mais une large partie de la population s'y est rendue de façon spontanée, comme s'il s'agissait de n'importe quel concert public.

Un deuxième profil correspond aux actions d'opposition. Il s'agit de manifestations qui critiquent la politique intérieure ou extérieure de la Fédération de Russie ou qui expriment leur solidarité avec les chefs de file des forces politiques se revendiquant « hors système » car non représentées à la Douma, chambre basse du parlement russe. La majorité de ces acteurs se considère comme les héritiers du mouvement des " rubans blancs» de l'hiver 2011-2012, qui réclamait l'organisation d' "élections honnêtes ». Ce signe de ralliement reste utilisé dans de nombreuses manifestations pour revendiquer l'appartenance à l'opposition démocratique ${ }^{5}$. Actuellement, les actions de ce type les plus importantes sont les marches organisées en mémoire de Boris Nemtsov, assassiné le 27 février 2015. Des hommages funèbres eurent lieu deux jours après sa mort à Moscou, à Saint-Pétersbourg et dans plusieurs autres villes, comme Vologda, Nijni-Novgorod, Kazan, Ekaterinbourg ou Sotchi. En 2016 et 2017, des défilés et des meetings furent à nouveau tenus le jour anniversaire de son assassinat ${ }^{6}$. Ces manifestations se heurtent souvent à des obstacles. En 2016, la demande d'autorisation présentée par des militants de Vologda fut rejetée par les autorités locales ; les organisateurs proposèrent alors la réunion d'un meeting devant le monument aux victimes des répressions politiques, sans faire explicitement référence à l'homme politique assassiné en 2015, et l'hommage put avoir lieu grâce à ce subterfuge. Les manifestations organisées par l'homme politique Alekseï Navalny et les courants qui s'en réclament sont également comptées parmi les manifestations

5. A. Trткоv, "Belaâ lenta 2011-2012 godov: političeskij simvol v povsednevnom okruženii [Le ruban blanc de 2011-2012: un symbole politique dans son environnement quotidien] ", in A. Arhipova, D. Radčenko et A. Trtkov (dir.), Gorodskie teksty i praktiki [Pratiques et textes urbains], vol. 1, Simvoličeskoe soprotivlenie [Résistance symbolique], Moscou, RANEPA, 2016, p. 69-84.

6. E. ÛGaJ, A. Krylova et A. Arhipova, " "Vyzov" i "maskirovka": političeskij aktivizm v malen'kom gorode [Le "défi " et la "dissimulation "; l'activisme politique dans une petite ville de province] ", in Gorodskie teksty i praktiki.., op. cit., p. 225. 
d'opposition. On peut citer le meeting des forces d'oppositions unies tenu le 20 septembre 2015 à Moscou, ainsi que les actions menées pour dénoncer les poursuites judiciaires dont Alekseï Navalny a fait l'objet. Il faut toutefois noter que les défilés les plus importants suscités par ce mouvement, marqués par une forte participation de la jeunesse, ont eu lieu en mars et juin 2017, dans le cadre d'une campagne pour dénoncer la corruption des élites russes; elles ne sont pas incluses dans cette étude dont le corpus a été clos à la fin de l'année 2016.

Un troisième type de manifestations est le fait de la "nouvelle gauche " d'une part, coalition hétéroclite se voulant une alternative démocratique au Parti communiste, lequel dispose d'élus à la Douma, et d'une fraction oppositionnelle des courants nationalistes d'extrême droite qui critique le régime de Vladimir Poutine et s'oppose à la guerre en Ukraine. À la différence des mouvements de contestation conjoncturels, les marches et les meetings qu'ils mobilisent se tiennent à dates régulières le plus souvent. Leur nombre n'a pas augmenté de façon significative au cours de la période étudiée. À gauche, il s'agit de la fête du $1^{\text {er }}$ Mai ; chez les nationalistes de droite, c'est la «Marche russe » organisée le 4 novembre ${ }^{7}$.

Un quatrième profil est constitué des meetings liés à des revendications sociales. On regroupe ainsi les actions menées par différents groupes comme les professionnels de santé, les chauffeurs routiers, les enseignants ou les retraités qui se sont activement mobilisés depuis 2014 autour de revendications catégorielles. Ainsi, les chauffeurs routiers ont commencé à susciter des actions de masse au début de l'année 2016 en réponse à un projet d'introduction de lourdes taxes sur le transport de marchandises empruntant les routes d'importance fédérale, un projet désigné sous l'acronyme "Platon». Leur action, qui a duré plusieurs mois, a renouvelé dans la société russe le stéréotype de "moujik rustre ", pour en faire une figure du mécontentement populaire à l'égard du régime, et non plus du soutien inconditionnel comme c'était le cas depuis quelques années ${ }^{8}$. Les revendications exprimées concernent la réduction des dépenses publiques dans le domaine de l'éducation ou de la santé, la suppression de bénéfices sociaux ou d'autres réformes de ce type. Elles ne s'inscrivent pas formellement dans un courant politique, mais véhiculent toutefois le plus souvent des revendications et des mots d'ordre politiques.

\section{Méthodologie : la manifestation de rue comme acte de communication}

Depuis 2011, notre groupe d'anthropologues, de folkloristes, de sociologues et de spécialistes de la culture étudie, par l'observation directe, les meetings, les marches et les piquets revendicatifs car ils constituent une dimension incontournable de la vie politique et sociale. Les premiers résultats de ces travaux ont été exposés dans l'ouvrage Anthropologie de la protestation sociale en Russie, années 2011-2012'; les

7. Le 4 novembre est la date anniversaire de la révolte populaire, menée par Konstantin Minine et Dmitrij Pojarski, qui chassa les armées polono-lituaniennes de Moscou en 1612, événement considéré comme l'avènement d'un sentiment national populaire alors que le trône était vacant ; la date a été célébrée jusqu'en 1917, elle a été restaurée comme une fête officielle de la Fédération de Russie en 2005 sous le nom de "Fête de l'unité nationale ». [NdT]

8. D. RadČEnko, "Soprotivlenie protestu: obsuždenie "Antiplatona” v moskovskoj probke [La résistance à la contestation : une discussion du mouvement "Antiplaton " dans un embouteillage moscovite] ", in Gorodskie teksty i praktiki..., op. cit., p. 174-186.

9. A. Arhipova et M. Alekseevskij (dir.), “My ne nemy!”..., op. cit. ; voir aussi A. Titkov, "Vosstanie kul'turnyh mehanizmov: protest kak âzykovaâ igra [La rébellion des mécanismes culturels : la protestation sociale comme jeu de langue] ", Sociologičeskoe obozrenie, vol. 15, n 2, p. 208-229. 
formes verbales et non verbales de la protestation sociale contemporaine en Russie sont examinées dans l'ouvrage collectif Résistances symboliques ${ }^{10}$.

Laction politique publique est abordée comme un acte de communication, au sens que lui donne Roman Jakobson ${ }^{11}$. Dans cette perspective, la communication implique, pour être opérante, qu'un destinateur adresse à un destinataire un message à l'aide de différents codes. Dans le cas qui nous intéresse, en se rendant à une manifestation, l'individu devient le destinateur d'un message politique exprimé sous une forme forte (explicite) ou faible (silencieuse). Le corpus étudié ici est constitué par les énoncés forts (textes de slogans, images ou artefacts) recensés au cours des années 2011-2016 dans plus de cent manifestations de rue de toutes tendances. Ces marches, ces meetings et ces piquets revendicatifs ont été observés à Moscou, à Saint-Pétersbourg, à Novossibirsk, à Sébastopol, à Vologda, à Kazan, à Toula ainsi que dans d'autres villes. Toute forme de manifestation de la protestation, que ce soit une broche à l'effigie de Staline ou une pancarte proclamant " Poutine démission! ", constitue pour nous des énoncés forts qui peuvent être médiatisés par l'intermédiaire d'un code verbal (des pancartes) ou visuel (des dessins), d'artefacts (des vêtements, des insignes, des jouets) ou d'actes (l'organisation d'une performance lors d'un meeting) et de toute expression combinant ces différents éléments. Un manifestant de 2011 portait une pancarte indiquant «Vous n’avez pas été élus par moi », effectuant ainsi, selon la terminologie retenue, un énoncé fort par l'intermédiaire d'un code verbal. Lors d'une manifestation de soutien à la révolution ukrainienne, une manifestante s'est présentée vêtue de jaune et de bleu (figure 1, reproduite dans le cahier d'illustrations couleurs, p. III), couleurs du drapeau ukrainien, constituant ainsi un énoncé fort par l'intermédiaire d'un code vestimentaire, soit d'un artefact. Le travail de notre groupe " Analyse du folklore contemporain » repose donc sur l'observation directe des différentes manifestations de rue, observations enregistrées à l'aide de la photographie et de la vidéo. Toutes les prises de vue d'énoncés forts sont systématiquement recensées dans une base de données intitulée Voices of protest ${ }^{12}$ qui permet de quantifier, pour chaque événement, le nombre de personnes exprimant un énoncé fort. Ainsi, quand lors d'une manifestation plusieurs dizaines de personnes arborent des pancartes identiques, chacune d'entre elles est comptabilisée comme un énoncé distinct. Cette démarche permet de quantifier l'usage des différents slogans et éléments symboliques. Toutefois, lors de la saisie dans la base de données, un dédoublonnement est opéré et une seule image de l'énoncé est conservée. Un numéro d'identification est attribué à chaque énoncé unique relevé et analysé. L'enquête a également reposé sur la conduite de brefs entretiens avec des manifestants pour expliciter la signification donnée à certains symboles ou slogans.

Pour analyser les énoncés forts qui utilisent le code du slogan que nous avons collectés, verbaux et non verbaux, nous avons utilisé les méthodes de la linguistique de corpus qui reposent sur un traitement statistique. Nous cherchons ainsi à déchiffrer les différentes « voix » de ces manifestations de rue à partir de l'ensemble des énoncés collectés.

10. A. Arhipova, D. Radčenko et A. Titkov (dir.), Gorodskie teksty..., op. cit.

11. R. Jаковson, "Closing statements: Linguistics and Poetics ", dans Style in langage, Cambridge, MIT Press, 1960, p. 353. Traduction française : "Linguistique et poétique », in Essais de linguistique générale, Paris, Éditions de Minuit, 1963. [NdT]

12. Le prototype de la base de données Voices of protest est hébergé par le Centre de recherche sur l'Europe centrale de l'Université de Brême. Son exploitation est soutenue par le Deutscher Akademischer Austauschdienst (DAAD) et la Deutsche Forschungsgemeinschaft (DFG) dans le cadre du programme DiscussData. 


\section{Le " paradigme historique » comme ressource interprétative}

Comme nous l'avons déjà souligné, les manifestants font de plus en plus fréquemment référence à l'histoire : sur 6595 énoncés forts uniques recensés lors de soixante-cinq manifestations politiques entre l'hiver 2011 et l'automne 2016, $18 \%$ (soit 1163 énoncés) comparent les événements contemporains avec le passé ou comportent des références historiques.

Au cours des manifestations de l'hiver 2011-2012, de $2 \%{ }^{13}$ à $6 \%{ }^{14}$ des slogans, seulement, font appel à l'histoire ; lors de cette première période des protestations de rue dans la Russie contemporaine, les références au passé ne jouent qu'un rôle marginal. Le recours aux références historiques augmente sensiblement à partir de 2014. En septembre de cette année-là, lors de la Marche de la paix organisée par l'opposition pour dénoncer la guerre en Ukraine, le passé est convoqué dans $12 \%$ des slogans. L'année suivante, en 2015, cette tendance s'observe également dans les manifestations qui soutiennent le pouvoir : lors de la marche du mouvement " anti-Maïdan ", $11 \%$ des slogans font appel à l'histoire; lors des actions en faveur du rattachement de la Crimée, 12 \% ; du côté de l'opposition, le passé est convoqué dans $9 \%$ des slogans énoncés lors de la marche en hommage à Boris Nemtsov. En 2016, le phénomène s'accentue lors de plus grandes manifestations : $18 \%$ lors de la marche anniversaire de la mort de Boris Nemtsov à Saint-Pétersbourg, $16 \%$ à Moscou (voir tableau 2, p. 147). Ainsi, au cours des années 2014-2015, la mémoire historique, notamment de la période soviétique, est redevenue " chaude ", pour reprendre la terminologie de Jan Assmann ${ }^{15}$. Les références au passé se sont multipliées, aussi bien dans le discours politique officiel que dans les énoncés vernaculaires qui font référence à la situation courante ${ }^{16}$. Les manifestants que nous avons observés utilisent donc l'histoire comme une ressource interprétative.

Les réactions au conflit armé déclenché en 2014 dans l'est de l'Ukraine constituent un exemple marquant d'utilisation du passé pour interpréter des événements présents. Toutes les prises de position sur le conflit, qu'elles soient d'ordre politique, moral ou émotionnel, ont cherché à l'« inscrire » dans un contexte historique particulier et ont choisi dans le passé des "universaux moraux » susceptibles de nourrir leur jugement ${ }^{17}$. La situation dans le Donbass a ainsi pu être présentée par certains comme une confrontation entre "nos alliés et les fascistes", autrement dit, comme un nouvel épisode de la Seconde Guerre mondiale ; par d'autres comme une

13. Ce chiffre correspond au meeting de l'opposition organisé à Moscou le 6 mai 2012, la veille de la cérémonie d'investiture de Vladimir Poutine ; cet événement a été désigné par la suite sous le nom de "manifestation de Bolotnaïa ", du nom de la place où elle s'était déroulée.

14. Ce chiffre correspond au meeting de l'opposition du 24 décembre 2011 à Moscou, organisé pour dénoncer la fraude lors des élections à la Douma d'État.

15. J. Assmann, Das kulturelle Gedächtnis: Schrift, Erinnerung und politische Identität in frühen Hochkulturen, Munich, C. H. Beck, 1992 ; traduction française : J. Assmann, La Mémoire culturelle. Écriture, souvenir et imaginaire politique dans les civilisations antiques, Paris, Aubier, 2010, p. 71 [NdT].

16. Plusieurs chercheurs ont souligné, au début des années 1990, que les controverses répétées liées à la mémoire historique étaient une caractéristique commune des anciens pays socialistes : R. S. Esbenshade, "Remembering to Forget: Memory, History, National Identity in Postwar EastCentral Europe ", Representations, $\mathrm{n}^{\circ}$ 49, 1995, p. 72-96 ; T. JudT, "The Past is Another Country: Myth and Memory in Postwar Europe ", Daedalus, vol. 121, no 4, 1992, p. 83-118.

17. J. C. Alexander, "On the social construction of moral universals: the "holocaust" from war crimes to trauma drama ", in The Meanings of Social Life: A Cultural Sociology, Oxford, Oxford University Press, 2003, p. 27-84. 
résurgence des opérations d'ingérence militaire menées par l'URSS puis la Russie en Hongrie en 1956, en Tchécoslovaquie en 1968, puis en Géorgie en 2008 ; d'autres groupes, enfin, ont choisi de lire ces événements comme une nouvelle péripétie de la confrontation "séculaire » opposant la Russie à l'Occident. Il ne s'agit là que de quelques exemples, l'histoire pouvant fournir de nombreux "codes interprétatifs » pour un même événement.

L'annexion de la Crimée en mars 2014 et l'assassinat de Boris Nemtsov en février 2015 ont également constitué des tournants dans la lecture de la situation politique. Pour reprendre la terminologie de Reinhart Koselleck, ces événements ont ouvert des «brèches dans l'horizon d'attente ${ }^{18}$ ». L'expérience d'événements inattendus "bouscule les futurs possibles délimités par les expériences vécues " et conduit à une réévaluation à nouveaux frais du passé. Parallèlement, l'événement construit un nouvel horizon d'attente qui entraîne de nouvelles représentations des possibles et du futur souhaités : "ainsi, une brèche momentanée dans nos attentes refaçonne la relation que ces deux mesures [l'expérience et l'attente] entretiennent ${ }^{19}$ ". Les bouleversements de la politique extérieure russe survenus en 2014 ont produit un tel effet : les représentants des différents courants d'opinion se sont mis à produire un grand nombre de textes qui cherchaient à "rééquilibrer " les attentes et l'expérience en les rapprochant d'éléments connus tirés du passé historique. Les manifestants convoquent ainsi des références qui leur servent de clé pour interpréter l'actualité.

C'est ainsi qu'en 2014 le mouvement connu sous le nom de « Régiment immortel » (Bessmertny polk) s'est largement diffusé. Cette initiative, qui remonte à 2013, est un défilé organisé le jour de la victoire, le 9 mai, dont les participants arborent les portraits des membres de leur famille qui ont combattu pendant la Seconde Guerre mondiale. De 2014 à 2016, cette manifestation a pris une autre ampleur. Les participants ne se contentent plus de brandir des portraits de combattants, ils défilent en uniformes militaires, montent des bivouacs, entonnent des chants militaires et encadrent des colonnes de faux prisonniers de guerre allemands. La manifestation est ainsi passée d'un simple acte commémoratif à une reconstitution de la guerre et des défilés de la victoire. À Saint-Pétersbourg en 2016, les participants au défilé du "Régiment immortel » scandent : "Nous sommes prêts à le refaire!» Pour de nombreux participants, ce slogan et cette façon d'incarner symboliquement l'armée des vainqueurs, à l'aide de portraits, de cartes postales ou d'uniformes d'époque, servent à la fois à entretenir la nostalgie d'un passé idéalisé et à s'approprier le nouveau contexte politique, constitué par la guerre en Ukraine et la mise en place de sanctions occidentales. Une manifestante âgée de 53 ans, interrogée sur ses motivations pour participer à la marche du "Régiment immortel » organisée à Kirichy (région de Léningrad), nous a répondu : «Fierté de la victoire et sentiment d'unité, non seulement avec ceux qui défilent à mes côtés, mais avec tout le pays : ça fait longtemps qu'on n’avait pas ressenti cela! » Un autre manifestant, lors d'une

18. R. Koselleck, "Erfahrungsraum und Erwartungshorizont - zwei historische Kategorien ", in ID., Vergangene Zukunft. Zur Semantik geschichtlicher Zeiten, Francfort-sur-le-Main, Suhrkamp, 1995, p. 349-377 ; traduction française : Le Futur passé : contribution à la sémantique des temps historiques, Paris, Éditions de l'EHESS, 2016 [NdT].

19. Ibid. 
marche organisée dans le district de Stavropol, au sud du pays, a répondu ainsi à la même question :

C'est un sentiment de fierté de faire cette marche de la victoire avec [le portrait de] mon grand-père. Car il a sacrifié sa vie pour moi, alors que je n'étais même pas né. C'est le sentiment que son sang coule encore dans mes veines et que cette Grande Victoire est aussi la mienne ! Le sentiment d'unité entre générations, le sentiment de grandeur de notre pays victorieux ${ }^{20}$.

L'instrumentalisation du passé est donc renforcée dans les situations où les manifestations de rue font suite à un événement inattendu qui rompt avec la routine politique ; on cherche alors à le "rapprocher du connu ", à l'inscrire dans des catégories familières. Les auteurs de slogans ont souvent recours à l'analogie pour faire des rapprochements avec des événements historiques tirés de sources diverses, des souvenirs scolaires de cours d'histoire aux émissions des grands médias, susceptibles d'entrer en résonance avec la mémoire collective et de faire sens pour le plus grand nombre. Ces contraintes expliquent le nombre limité d'événements historiques sollicités pour construire ces analogies.

Plusieurs slogans utilisés dans les manifestations du mouvement "antiMaïdan ", par le NOD ou les meetings de soutien au rattachement de la Crimée, font référence à des épisodes à leurs yeux "sacrés " de l'histoire russe : la période de la Rous' kiévienne (la "Sainte Russie ") et de l'Empire russe (mentionnée dans $5 \%$ de l'ensemble des slogans utilisant des références historiques) ou la victoire de 1945 (7 \%). S’y ajoutent des périodes sombres qui jouent à leurs yeux le rôle de repoussoir : la perestroïka et la chute de l'URSS en 1991 (10,6 \%). Au contraire, les manifestants d'opposition comparent les événements ukrainiens avec les interventions militaires soviétiques dans les démocraties populaires $(2 \%)$ ou la guerre en Afghanistan (1,5\%). Des références sont faites également aux répressions staliniennes (4\%), aux assassinats ciblés et répressions politiques menés depuis 1991 (4\%), ainsi qu'aux grandes figures de la dissidence, Andreï Sakharov et Vaclàv Havel (3\%).

Deux thèmes sont sollicités indifféremment par les groupes loyalistes et par l'opposition : les adversaires politiques sont rapprochés des "nazis" $(5 \%)$ et les dirigeants politiques sont comparés à Staline (3\%), figure qui est convoquée soit comme tyran, soit comme modèle exemplaire. Une pancarte observée le $1^{\text {er }}$ mai 2015 mentionne ainsi :

Sous son régime, on travaillait dans l'honneur,

Pas un voleur fainéant,

Et tout le pays avait suffisamment d'argent.

Après lui, les caisses sont vides les enfants,

Le fruit de notre labeur va dans les poches des oligarques

Les références aux révolutions de 1917 et à la guerre civile ne sont pas très fréquentes ( $4 \%$ ), quelle que soit l'orientation politique de la manifestation. Les autres

20. A. Arhipova, D. Doronin, A. Kirzûk, D. Radčenko, A. Sokolova, A. Titkov et E. ÛGaj, "Vojna kak prazdnik, prazdnik kak vojna: Performativnaâ kommemoraciâ dnâ pobedy [La guerre comme une fête, la fête comme une guerre : les commémorations performatives du jour anniversaire de la victoire] ", Antropologičeskij forum, n 33, 2017, p. 84-122. 
événements de l'histoire russe ne sont utilisés que de façon exceptionnelle. Enfin, il faut noter par ailleurs que certains slogans historiques ne renvoient pas à des événements précis mais font référence à l'" inviolabilité de l'histoire " - on trouve des pancartes appelant à "Ne pas réécrire l'histoire " dans les manifestations de toutes tendances.

Dans l'ensemble du spectre politique, les références au passé servent ainsi de cadre interprétatif pour conférer du sens au présent et donnent naissance, dans la confusion, à un nouveau " langage historique " qui possède sa grammaire et sa sémantique propres.

\section{La grammaire des paradigmes historiques ou comment les manifestations parlent du passé}

Depuis deux ans, le discours des manifestations de rue produit donc un grand nombre d'énoncés qui incluent un paradigme historique. Le rapport entre le présent et le passé peut être effectué par le destinateur selon différentes modalités ; l'enquête menée a permis de dégager cinq grands types d'énoncés à caractère historique qui constituent, en quelque sorte, la "grammaire" de ce langage politique (tableau 1).

La modalité la plus simple est le constat d'une similarité entre un événement présent et un fait du passé ; par exemple, le manifestant qui énonce «2016, c'est un nouveau 1937 ». Puisqu'il s'agit de décrire la réalité à l'aide d'une analogie, nous appelons ce mode descriptif. Le rapprochement peut également avoir pour fonction d'exprimer une volonté de transformer ou de consolider une situation, de dire qu'on ne souhaite pas qu'une situation historique connue se répète; nous qualifions alors cet énoncé d'optatif. Une autre modalité correspond aux invocations adressées à des figures historiques ou des personnages célèbres - énoncés que nous qualifions de vocatif. Un dernier type correspond aux formules qui, sans exprimer un souhait ou une analogie, constituent un acte performatif, au sens que John Austin donne à ce terme, car elles expriment un jugement de valeur ou une émotion destinés à modifier la perception de l'événement et non simplement à le décrire ou à communiquer un point de vue ${ }^{21}$. C'est le cas par exemple d'une pancarte observée à Moscou en novembre 2016 dans un piquet revendicatif, qui proclamait : "En novembre 1956, l'URSS écrasait avec ses chars la révolution en Hongrie. J'ai honte" (figure 2, voir p. III). Cette formule fait sens au regard du refus des autorités soviétiques et des autorités russes qui lui ont succédé de condamner cette intervention ; en exprimant ainsi une condamnation et un sentiment de honte à la place du gouvernement, le manifestant effectue donc un acte performatif.

La distinction entre ces quatre modes d'énoncés est parfois ténue, mais elle correspond à une gradation dans la volonté de transformation de la réalité : d'une simple comparaison descriptive à l'utilisation d'une référence historique pour énoncer un jugement, voire pour réaliser un acte de langage. La typologie que nous avons élaborée ne repose donc pas strictement sur des éléments grammaticaux, mais sur une analyse des présupposés mis en œuvre par le destinateur et qui peuvent être facilement compris par l'auditoire. Cette méthode d'analyse du discours a été

21. J. Austin, How to do Things with Words: The William James Lectures delivered at Harvard University in 1955, Cambridge, Harvard University Press, 1962 ; traduction française : Quand dire, c'est faire, Paris, Éditions du Seuil, 1991. 
largement utilisée dans les études pragmatiques et cognitivistes des années 19601980 : au-delà du "sens superficiel " qui résulte de la somme des mots rassemblés dans un énoncé, il existe un sens second, non verbal, que le destinateur élabore à partir de sa compréhension du contexte ${ }^{22}$. Par exemple, lors du concert-meeting organisé en soutien au rattachement de la Crimée, douze mois après son annexion et la mise en place des sanctions occidentales, on pouvait voir une pancarte avec l'inscription : "Roosevelt et Churchill étaient plus malins " (figure 3, voir p. IV). La signification du message ne repose pas sur les seuls termes utilisés ; elle implique de déterminer avec qui la comparaison est faite, a priori, le président Obama. Mais il reste à comprendre le sens politique de ce slogan et le rapport établi entre les figures de Churchill et Roosevelt et la situation ukrainienne. Le destinataire doit donc faire appel à sa connaissance de la conférence de Yalta qui a rassemblé en Crimée Staline, Roosevelt et Churchill en 1945. Ainsi, le présupposé du slogan invite le président Obama, sans le nommer, à imiter son prédécesseur et à entamer des négociations plutôt que d'entrer en conflit avec la Russie. Il s'agit donc, en d'autres termes, d'une forme complexe d'énoncé sur le mode optatif.

Nous avons encore identifié un cinquième type d'énoncés, nettement distinct des précédents, le mode réflexif. Il s'agit d'assertions qu'on peut qualifier de métahistoriques, qui ne cherchent pas à transformer le présent par une comparaison avec le passé, mais à dénoncer l'instrumentalisation politique de l'histoire.

Examinons dans l'ordre chacun de ces modes de référence à l'histoire, en s'attachant à des exemples complexes.

\section{Les énoncés descriptifs : l'explication du présent par le passé}

Ce mode est le plus fréquent, il constitue 71,6 \% des slogans utilisant une référence historique (tableau 1, p. 147). Il s'agit bien entendu des énoncés les plus simples. Ils reposent sur l'utilisation d'un répertoire limité d'événements historiques qui sont chacun connotés positivement ou négativement et permettent de qualifier par analogie un événement contemporain. On observe plusieurs modèles de construction de la comparaison.

L'opération la plus simple consiste à créer une relation entre deux événements à partir d'un ou de plusieurs critères communs. On peut donner comme exemple la pancarte observée lors de la marche en hommage à Boris Nemtsov de 2015, où figurait le slogan "Nadia d'Arc ». Un parallèle était ainsi établi entre Nadejda Savtchenko ${ }^{23}$, qui effectuait une grève de la faim à la date de la manifestation, et Jeanne d'Arc. Le rapprochement entre les deux personnages est fondé sur des points communs : il s'agit de femmes qui revendiquent un rôle masculin dans les combats, font preuve d'héroïsme, incarnent la résistance à l'envahisseur, sont prisonnières et victimes d'une justice partisane, prennent la valeur de martyres, etc.

22. G. Lakoff, Women, Fire, and Dangerous Things: What Categories reveal about the Mind, Chicago, University of Chicago Press, 1987.

23. Nadejda Savtchenko est une femme officier de l'armée de l'air ukrainienne, médaillée pour sa bravoure. En été 2014, elle a été conduite dans des circonstances inconnues sur le territoire de la Fédération de Russie et condamnée par un tribunal russe à vingt-deux ans d'emprisonnement pour sa responsabilité alléguée dans la mort de journalistes russes présents sur le front ukrainien. De nombreuses voix de l'opposition en Russie ont dénoncé ce verdict comme illégal et organisé des piquets et des manifestations pour la défendre. Nadejda Savtchenko a finalement été graciée par un décret présidentiel le 25 mai 2016. 
C'est la réception du destinataire qui est susceptible de mettre l'accent sur l'un ou l'autre de ces éléments. Sa lecture repose sur l'équivalence morale et émotionnelle qui est attribuée à une figure historique considérée comme héroïque et un personnage contemporain qui bénéficie ainsi de son prestige. Ce type de rapprochement peut également servir à opposer des événements historiques contradictoires. Lors d'un piquet pacifiste organisé à Ekaterinbourg le 27 septembre 2015, une pancarte opposait ainsi l'histoire récente de l'Ukraine et les conflits armés dans lesquels la Fédération de Russie s'était trouvée engagée depuis 1991 :

$$
\begin{aligned}
& \text { Ukraine : } 22 \text { ans sans conflits } \\
& \text { Russie : } 1992 \text { - Transnistrie } \\
& \text { 1994-1996 - Tchétchénie } \\
& \text { 1999-2000 - Tchétchénie } \\
& \text { 2009- Ossétie du Sud et Abkhazie. }
\end{aligned}
$$

Le plus souvent, le destinataire du message doit interpréter le sens du slogan en reconstituant les présupposés de l'énoncé à partir de sa connaissance du contexte, de sa culture historique et, bien sûr, du cadre de la manifestation à laquelle il participe. Ainsi, un slogan utilisé lors du concert-meeting organisé en soutien au rattachement de la Crimée proclamait : "1991-2014 : le troisième siège de Sébastopol ». La référence historique est implicite, la période d'administration de la ville portuaire de Crimée par l'Ukraine étant assimilée aux sièges de Sébastopol de 1854-1855 et de 1941-1942. Ces derniers sont dans la mémoire collective les symboles d'une résistance russe acharnée en dépit de la supériorité des forces de l'adversaire : les armées anglo-françaises au XIX siècle, hitlériennes au $\mathrm{XX}^{\mathrm{e}}$ siècle et donc, implicitement, ukrainiennes au XXI' (figure 4, voir p. V).

On retrouve un procédé proche dans ce slogan de la Marche de la paix moscovite du 21 septembre 2014, avec une référence implicite à la déroute russe face au Japon au début du $\mathrm{XX}^{\mathrm{e}}$ siècle : «Une petite guerre victorieuse pour rehausser le prestige ? On a déjà tenté le coup en $1905^{24}$ ”.

\section{Les énoncés optatifs : désirs de recommencement, de changement ou de défense de l'existant}

Les énoncés optatifs et vocatifs sont d'une structure plus complexe et reposent sur des présupposés plus nombreux. Lorsqu'une figure historique est convoquée, il faut que le message soit lisible par les autres manifestants aussi bien que par le reste du public; il faut donc qu'il soit facilement identifié et interprété par le plus grand nombre. Ainsi, si la victoire de l'URSS en 1945 est jugée positivement par la quasitotalité de l'opinion publique, la perspective d'un nouveau conflit international, même victorieux pour la Russie, suscite des réactions évidemment plus complexes. Une figure historique célèbre, si elle est facilement identifiée, peut être appréciée de façon divergente. Ainsi, Boris Eltsine peut incarner la démocratie pour certains, l'effondrement du pays ou la crise économique pour d'autres.

24. En 1904, le ministre de l'Intérieur Viatcheslav Plehve aurait eu les propos suivants pour évoquer la perspective d'un conflit avec le Japon : «Pour étouffer la Révolution, il nous faut une petite guerre victorieuse ». L'expression est devenue une formule consacrée en Russie pour décrire une situation de tension politique intérieure que le pouvoir tente de résoudre par l'intermédiaire d'une crise internationale. 
Les énoncés optatifs sont ainsi moins fréquents (12,6\% du corpus). Ils utilisent la référence au passé pour exprimer une volonté de changement ; l'histoire étant utilisée comme un répertoire d'exemples de lois sociales susceptibles d'agir au présent. Les références historiques sont convoquées pour lire l'actualité et se projeter dans l'avenir. Le slogan, déjà cité, "Nous pouvons le refaire ", sous-entend ainsi qu'un conflit international avec une issue victorieuse pour la Russie est possible, comme en 1945, mais aussi souhaité par les manifestants. Comme cela a déjà été dit, la valeur optative de ces énoncés n'est pas nécessairement déterminée par la syntaxe utilisée, mais dépend des présupposés convoqués.

Les slogans optatifs les plus fréquents sont ceux qui menacent un adversaire supposé. Par exemple, lors des tensions qui suivirent la destruction d'un avion de chasse russe par l'armée turque, le slogan suivant fut utilisé lors d'une manifestation organisée devant l'ambassade de Turquie à Moscou :

5 victoires légendaires de l'armée russe sur la Turquie :

- 1770 : bataille de Tchesmé, amiral Ouchakov

- 1787 : prise d'Izmaïl, Souvorov

- 1853 : prise de Sinope, amiral Nakhimov

- 1877 : bataille de Cheïnovo

- 1914 : prise de Sarikamis

$-2015: ?^{25}$

Des slogans se présentent sous la forme d'une simple succession de chiffres, comme celui-ci : "1612-1812-2012». Le destinataire doit alors identifier ces dates et le sens qui leur est donné, en s'appuyant sur sa connaissance du contexte. L'exemple donné ici a été relevé lors d'une marche nationaliste et s'apparente à une tentative de démonstration "numérologique " : à 200 ans d'intervalle, le Kremlin a été libéré d'envahisseurs lituano-polonais puis français, les manifestants proclament ainsi leur volonté de vaincre les ennemis qui s'attaqueraient à la Russie en 2012. On peut également rapprocher de ce modèle les slogans qui appellent à la restauration d'une situation passée ou à une forme de compensation historique pour réparer une situation considérée comme une injustice historique. Un exemple sommaire de ce type d'énoncé fait référence aux pertes territoriales de la Russie; lors de la marche "anti-Maïdan" de 2015, une pancarte proclamant "Maintenant, il faut nous rendre l'Alaska " a ainsi été observée.

Les énoncés optatifs ont été utilisés avec une grande fréquence dans les manifestations d'opposition en 2011 (50\% des slogans utilisant des références historiques), les participants réclamant alors un changement rapide de la situation politique. Pendant la crise de l'année 2014 qui suivit l'annexion de la Crimée, dans un contexte de tensions internationales et de difficultés liées aux sanctions économiques visant la Russie, l'usage des énoncés optatifs est resté assez fréquent (27\%) avant de diminuer fortement les années suivantes (tableau 4, p. 148).

Un usage notable des énoncés optatifs a été recensé dans un seul type de manifestations loyalistes, les concerts-meetings organisés pour célébrer le rattachement de la Crimée. Ils se présentaient sous la forme de slogans imprimés en plusieurs exemplaires sur des pancartes, en imitant la forme manuscrite. Par ailleurs, lors de

25. Pancarte du meeting tenu devant l'ambassade de Turquie à Moscou le 26 novembre 2015. 
ces événements, il n'est pas rare que le même slogan soit répété sous diverses formes par différents manifestants. Ainsi, lors du meeting de 2015, on a pu observer le slogan suivant, utilisé à la fois sous forme manuscrite et en caractères d'imprimerie : «Obama! Prends exemple sur Roosevelt : viens à Yalta négocier!" (figures 5 et 6 , voir p. V). Malgré l'usage de l'impératif, il s'agit bien d'un énoncé optatif, proposant de répéter la conférence de Yalta en 1945, considérée comme un succès diplomatique de l'URSS - cette fois explicite, à la différence du slogan isolé précédemment évoqué. La situation militaire et diplomatique de l'époque est implicitement transposée en 2015 : l'Ukraine occupe la position de vaincue de l'Allemagne nazie ; Obama est invité à discuter avec une Russie qui serait en position de force, comme l'URSS à la fin de la guerre, Poutine étant supposé jouir du prestige de Staline. Le slogan prend une force supplémentaire en pointant le site de Yalta, ville de Crimée récemment "libérée ». Cet usage isolé de l'optatif dans les manifestations loyalistes et l'usage de la forme manuscrite donnent l'impression d'une mise en scène particulière, soulignant que ce souhait serait émis de façon unanime par la voix du peuple.

\section{Les énoncés vocatifs}

Ils constituent $13,3 \%$ du corpus. Il s'agit des énoncés qui invoquent des personnages historiques - acteurs politiques, de l'art ou de la culture. Ainsi, plusieurs pancartes des meetings d'opposition de 2011 figuraient une photographie d'Andreï Sakharov avec le slogan «Andreï Dmitrievitch avec nous!». Il s'agissait à la fois de convoquer la figure de cet intellectuel soviétique, défenseur des droits de l'homme et porte-voix de la dissidence, mais également d'une allusion au fait que la manifestation se déroulait sur une avenue de la capitale qui porte son nom. On rencontre également des formes d'invocation indirecte. Lors des marches rendant hommage à Boris Nemtsov, on a observé plusieurs pancartes comportant des citations de ses articles, jouant ainsi sur sa présence et une prise de parole virtuelle au cours de la manifestation. Invoquer le nom ou arborer le portrait d'une personnalité produit une participation symbolique dans les manifestations et une adhésion aux mots d'ordre.

\section{Les énoncés performatifs}

Les énoncés performatifs impliquent un engagement individuel plus fort du destinateur ; il s'agit donc de slogans plus rares qui constituent seulement $1,5 \% \mathrm{du}$ corpus. Le paradigme historique est utilisé sous une forme qui en fait un acte de langage. L'énoncé repose sur une description où le destinateur qualifie négativement une situation politique à l'aide d'une analogie historique et réalise par ailleurs ce qu'il énonce en exposant sa position à l'égard de ces événements, par exemple l'expression d'une excuse publique (figures 2 et 7 , voir p. III et V).

Les énoncés performatifs sont ainsi principalement les expressions d'une émotion politique (figure $7, \mathrm{p} . \mathrm{VI}$ ). Ces slogans reposent sur le présupposé qu'un individu peut prendre sur lui la responsabilité d'un événement passé auquel il n'a pas participé directement et présenter des excuses au nom d'un groupe. En exprimant sa honte pour les interventions militaires soviétiques et russes en territoire étranger, la pancarte observée à la Marche de la paix réalise plusieurs opérations : expression d'un désaccord avec ces initiatives des gouvernements successifs, désolidarisation avec une politique menée au nom des intérêts de la Russie, mise à distance d'événements subis sans pouvoir agir sur eux directement. Le nombre de ces formes d'expression a 
quadruplé en 2014 (tableau 4, p. 148), notamment lors de la Marche de la paix suscitée en réaction à la guerre avec l'Ukraine, un "pays frère ", qui choque une grande partie de l'opinion. L'émotion des manifestants était si forte qu'ils ne se contentaient pas de décrire ou de dénoncer la situation à l'aide de références historiques, mais de la disqualifier ou de s'en désolidariser avec le plus de force possible.

On recense également des énoncés performatifs dans les manifestations loyalistes, avec une fréquence similaire à celle des meetings d'opposition (tableau 3, p. 148). Ces derniers jouent alors sur le sentiment de fierté pour l'histoire nationale. Cette exaltation patriotique s'exprime à l'égard d'événements historiques, comme le slogan "Je suis fier de la Victoire de 1945 », ou contemporains, comme la pancarte observée au concert-meeting de 2015 : «Je suis fier des habitants de la Crimée ! Je suis fier de mon pays!" (figure 8, voir p. VII). On peut lire dans ces expressions une forme de compensation face à un sentiment d'insécurité, ainsi qu'une forme de mobilisation patriotique destinée à défendre la Russie des attaques dont elle pourrait faire l'objet de la part des pays occidentaux.

Si les énoncés performatifs exprimant la culpabilité sont caractéristiques des manifestations de l'opposition et de l'affirmation de la fierté spécifique aux actions loyalistes, ils sont absents des mouvements liés à des revendications sociales (tableau 3, p. 148).

\section{Les énoncés réflexifs}

La fréquence d'utilisation du paradigme historique dans les manifestations de rue conduit à l'observation d'énoncés d'ordre "métahistorique " qui réagissent à cette exploitation politique du passé. Un petit nombre de slogans mettent ainsi en question la valeur de l'histoire et les interprétations qui en sont faites : "Nous n'avons pas besoin d'une histoire alternative ${ }^{26}$ "; " Je veux apprendre l'histoire, pas de la propagande - en cours d'histoire : "les Russes n'ont jamais agressé militairement personne" 27 ".

\section{Différenciation des usages politiques des références historiques}

Ces cinq modes d'énoncé d'un paradigme historique sont-ils présents dans des proportions équivalentes dans les différents types de manifestations? On observe un recours croissant aux références historiques dans tous les types de manifestations depuis 2011, à l'exception des actions partisanes des mouvements de gauche ou de droite (tableau 2, p. 147). On rassemble pourtant dans cette dernière catégorie des mouvements politiques très différents, communistes, monarchistes, néofascistes, etc., qui ont en commun de s'inscrire dans une tradition politique ancienne et de défendre des projets politiques inspirés de modèles passés. Pourtant, ces manifestants expriment leurs positions politiques à l'aide de symboles partisans, comme les drapeaux, les croix gammées, les faucilles et les marteaux, plutôt qu'à travers des slogans particuliers.

26. Pancarte observée lors d'une manifestation du mouvement NOD, 28 avril 2016. Les militants protestaient ainsi contre l'organisation d'un concours scolaire consacré à l'histoire familiale des élèves.

27. Slogan observé lors du meeting organisé en défense de la fondation "Dynastie " à Moscou, le 6 juin 2015. La fondation était consacrée à la publication d'ouvrages de vulgarisation destinés à valoriser la recherche et soutenir l'enseignement. 
Les manifestations liées à des revendications sociales sont également, à première vue, faiblement utilisatrices du paradigme historique dans leurs énoncés. De fait, dans $40 \%$ des manifestations de ce type que nous avons observées, aucune référence historique n’a été relevée. Ceci les distingue fortement des autres manifestations : les slogans faisant référence au passé ne sont absents que de $8 \%$ des manifestations d'opposition analysées et nous n'avons rencontré aucune manifestation partisane ou loyaliste passant l'histoire totalement sous silence. En outre, les références historiques utilisées dans ce type de manifestations sont très peu diversifiées : la moitié des énoncés expriment la volonté de restaurer ou de protéger un héritage du passé, que ce soit le système de protection sociale soviétique ou des monuments du patrimoine architectural.

Le recours croissant au paradigme historique dans les manifestations repose donc principalement sur les actions organisées par l'opposition et les groupes loyalistes. Ces derniers ont une grande inclination pour l'utilisation des références historiques, tendance de plus en plus marquée au cours de l'année 2016, à la différence des manifestations d'opposition. Ce phénomène s'explique par la combinaison de deux facteurs. En premier lieu, pour prendre une expression empruntée à la psychologie, on observe un phénomène de "renforcement positif » en 2014 et 2015 : l'auditoire des manifestations loyalistes réagit dans son ensemble très favorablement aux parallèles historiques utilisés pour donner sens à la guerre en Ukraine. En 2014, pendant la phase la plus aiguë du conflit, le discours prorusse et progouvernemental des médias a été saturé de références à la Seconde Guerre mondiale, dont la présence était physiquement et matériellement intégrée à la présentation des événements : récit de la découverte de dépouilles de soldats soviétiques lors du creusement de tranchées dans la région de Donetsk, utilisation par les combattants des républiques populaires de Donetsk et de Lugansk de chars T-34 et Joseph-Staline-3 qui avaient été retirés des monuments aux combats de $1945 \ldots$ Toutes ces informations nourrissent l'idée que "l'histoire est de notre côté " et suscitent l'enthousiasme des partisans de la sécession du Donbass. On peut souligner, d'autre part, que le discours politique des forces loyalistes est dépourvu de mots d'ordre renvoyant à l'avenir ; les références au passé soviétique et impérial russe sont donc largement mobilisées par les manifestants. Celles-ci prennent la forme d'énoncés descriptifs, bien entendu, mais également de formes optatives (tableau 3, p. 148). Là encore, cette situation s'explique par la fréquence des arguments d'autorité empruntés au passé dans le discours politique officiel ; les manifestants loyalistes se réfèrent ainsi de façon privilégiée au précédent de la victoire de 1945.

Les militants du Mouvement de libération nationale (NOD) constituent le noyau des meetings loyalistes étudiés. L'objectif affiché de ce groupe est de « restaurer la souveraineté " de la Russie, qui aurait été " perdue » en 1991, avec la disparition de l'URSS. Le passé soviétique et tout particulièrement la " Grande guerre patriotique » constituent donc pour ce mouvement une ressource symbolique majeure ${ }^{28}$. Celle-ci nourrit les slogans et alimente également de nombreux énoncés non verbaux, sous la forme d'artefacts ou de mises en scène. Lors de leurs manifestations, les militants

28. L'expression "Grande guerre patriotique », calquée sur l'appellation de la " Guerre patriotique » de 1812 (contre Napoléon), est depuis l'époque stalinienne utilisée en russe pour désigner la Seconde Guerre mondiale, ou plus précisément la phase du conflit impliquant le territoire de l'URSS entre l'invasion par les armées hitlériennes en juin 1941 et la victoire sur l'Allemagne en mai 1945. [NdT] 
du NOD sont vêtus avec des uniformes de l'Armée rouge et entonnent des chants militaires soviétiques, accompagnés d'un accordéon : ce type de démonstration peut être interprété de différentes façons. Il s'agit d'un emprunt à la pratique des défilés de masse qui constituaient un rituel politique de la période soviétique. On peut également y voir la citation d'une image célèbre dans l'opinion russe, celle du "soldat soviétique chantant dans les rues de Berlin au son de l'accordéon ", rendu populaire par plusieurs photographies de mai 1945. Les chants soviétiques entonnés dans les rues de Berlin en 1945 étaient une façon de souligner symboliquement la victoire sur la capitale allemande. La répétition de cette scène dans les rues de Moscou en 2015 n'est pas une simple citation de cette image historique célèbre, mais une mise en scène qui implique ses acteurs et ses spectateurs dans une réactualisation des scènes de victoire et accomplit ainsi un désir de se présenter comme des vainqueurs (figure 9, voir p. VIII). Nous avons qualifié de " commémoration performative ${ }^{29}$ " cette forme d'incarnation matérielle de la mémoire. Ce type de "souvenir par la répétition ${ }^{30}$ " se développe activement depuis 2014, devenant une façon parmi d'autres d'organiser une manifestation du NOD, aussi bien qu'une fête municipale le 9 mai, jour de la célébration de la victoire sur l'Allemagne nazie.

L'utilisation d'artefacts et de modèles de comportements empruntés à la " guerre sacrée " dans ces commémorations performatives donne aux militants loyalistes une justification pour recourir à la violence symbolique, verbale et même, dans des cas extrêmes, physique. Ainsi, au printemps 2016, des militants du NOD, habillés en uniformes des années 1940 et arborant une copie de l' " étendard de la Victoire ${ }^{31}$ ", ont aspergé de " teinture verte ${ }^{32}$ " les finalistes du concours "L'individu dans l'histoire " organisé par l'association Memorial, dénoncée comme une "cinquième colonne 33 ". La défense de symboles historiques sacralisés légitime ainsi le recours à la violence.

Dans les manifestations de l'opposition, le paradigme historique est également fréquemment sollicité en 2014 et 2016, mais dans des proportions moindres que ce qui a pu être observé du côté des loyalistes.

En 2014, l'augmentation du nombre d'énoncés historiques du côté de l'opposition s'explique par le choc qu'ont constitué la guerre en Ukraine et le rattachement de la Crimée à la Fédération de Russie. Ces deux événements ont provoqué une brèche dans l' " horizon d'attente " de cette frange de l'opinion ${ }^{34}$, qui nécessitait d'élaborer un modèle interprétatif adapté, d'autant que le discours médiatique était marqué par une forte ambivalence : s'agissait-il d'une guerre civile ou d'actes

29. A. Arhipova, D. Doronin, A. Kirzûk, D. Radčenko, A. Sokolova, A. Titkov et E. Ûgaj, "Vojna kak prazdnik ", art. cité.

30. S. A. Oushakine, "Remembering in Public: On the Affective Management of History ", $A b$ Imperio, $\mathrm{n}^{\circ} 1,2013$, p. 269-302.

31. L'étendard de la Victoire (Znamia Pobedy en russe) désigne la bannière de régiment de l'Armée rouge qui fut dressée au sommet du Reichstag le $1^{\text {er }}$ mai 1945. [NdT]

32. La "teinture verte" (zelenka) est un antiseptique d'usage courant en Russie au fort pouvoir colorant, utilisé fréquemment depuis 2010 pour attaquer et humilier en public des opposants politiques. [NdT]

33. L'association de défense des droits de l'homme "Memorial » mène des actions pédagogiques et scientifiques consacrées à la mémoire des victimes des répressions politiques de la période soviétique. Elle est actuellement considérée par la législation russe comme un " agent de l'étranger » en raison d'une partie de ses sources de financement.

34. R. Koselleck, "Erfahrungsraum und Erwartungshorizont...", art. cité. 
terroristes ? D'une affaire intérieure ukrainienne ou d'une guerre entre la Russie et l'Ukraine qui ne disait pas son nom ? Les analogies historiques permettaient ainsi de construire un discours d'opposition, inscrivant les événements en cours dans l'héritage de la politique impériale et coloniale de la Russie.

Le nouveau pic de références historiques observé en 2016 est marqué par le recours important aux énoncés vocatifs (tableau 4, p. 148). L'invocation de figures tutélaires est bien entendu une constante des manifestations d'opposition tout au long de la période étudiée. Lors des manifestations de la place Bolotnaïa et de l'avenue Sakharov en 2011, on relevait des références à des personnalités très variées, que ce soit des écrivains comme Alexandre Herzen (1812-1870) et Sergueï Dovlatov (1941-1990), le Mahatma Gandhi ou Andreï Sakharov. En 2014, en revanche, les personnalités invoquées étaient presque exclusivement des grands noms de la littérature. Après l'assassinat de Boris Nemtsov en 2015, toutefois, son portrait et ses citations sont devenus une ressource symbolique centrale qui en a fait une sorte de "Lénine de l'opposition ». Les marches d'hommage à sa mémoire sont les manifestations d'opposition qui ont rassemblé le plus grand nombre de participants. L'invocation de Boris Nemtsov est ainsi un moyen d'exprimer son appartenance politique lors d'une manifestation. Parallèlement à cette progression des références aux figures d'autorité, les formulations optatives ont baissé sensiblement. Alors qu'elles représentaient $50 \%$ des énoncés à caractère historique en 2011 , elles ont pratiquement disparu en 2016. La nostalgie historique semble être désormais associée aux seuls slogans loyalistes.

\section{Un « nouveau langage » pour décrire une réalité nouvelle}

La contestation de l'hiver 2011-2012 visait à changer le cours politique et à transformer l'avenir en réclamant la révision des résultats des élections législatives, en tentant de contrecarrer la réélection de Vladimir Poutine à la présidence de la Fédération de Russie et en dénonçant les contradictions entre l'impartialité revendiquée de l'administration et son instrumentalisation politique. Ainsi, la majorité des pancartes entendaient dénoncer ou défendre des personnalités politiques, en somme, à réclamer des changements dans la vie politique. En 2012-2013, ce langage protestataire a volé en éclats. La majorité des participants du mouvement contestataire de 2011-2012 ont alors perdu espoir dans la possibilité de réformer le régime, certains considérant même que s'opposer est devenu dangereux. Comme le déclare une manifestante interrogée en 2013, qui avait participé au mouvement des " rubans blancs ", en faisant allusion à Vladimir Poutine : "Tout n'a fait qu'empirer, parce que nous l'avons énervé ${ }^{35}$ ". Par ailleurs, le contexte politique a sensiblement changé. La situation géopolitique et sociale complexe des années 2014-2016, liée à l'annexion de la Crimée, au conflit ukrainien, aux sanctions économiques et à la guerre en Syrie, suscite des interprétations opposées des différentes forces politiques. C'est pourquoi s'affirme alors une attente pour un " nouveau langage " revendicatif, susceptible de rendre compte de la nouvelle situation politique. Les manifestants ressentent le besoin de s'appuyer sur des figures reconnues ou des références historiques pour exprimer leur sensibilité politique. Les références au passé sont ainsi

35. Entretien avec A.A., habitante de Moscou âgée de 48 ans, participante des manifestations de 2011-2012, enregistré par Alexandra Arkhipova. 
convoquées de façon plus fréquente et constituent une part importante des énoncés recensés dans les manifestations, en lien avec le tournant géopolitique que connaît le pays à partir de 2014 .

Toutes les manifestations mobilisent alors une même forme de paradigme historique. Les événements courants sont rapprochés avec des moments de l'histoire extérieure de la Russie, tels que les guerres, les conflits internationaux et les rivalités entre puissances. La période soviétique constitue le noyau des références utilisées par les manifestants des différents courants politiques, qu'il s'agisse de la période stalinienne ou de l'effondrement de l'Union soviétique en 1991, celle-ci étant représentée tantôt comme " empire du mal ", tantôt comme "État fort ". Les énoncés recensés ne comportent presque aucune référence aux conflits intérieurs, que ce soit la révolution, la guerre civile de 1918-1922, ou les mouvements de contestation de la seconde moitié du XX $\mathrm{XX}^{\mathrm{e}}$ siècle tels que la dissidence. L'exploitation politique de l'histoire s'exerce avec la plus grande intensité dans les pôles opposés que constituent les mouvements loyalistes et les courants d'opposition. Ces groupes sont ceux qui ressentent le plus la nécessité d'expliquer les événements présents et d'attirer la sympathie de l'opinion en ralliant à leur cause des figures d'autorité morale tirées de l'histoire. Toutefois, leurs stratégies d'exploitation des références historiques different sensiblement.

Les courants loyalistes n'ont pas besoin de chercher des figures d'autorité parmi les personnages historiques car ils s'appuient sur les dirigeants politiques au pouvoir. Mais ils cherchent à légitimer leur mouvement, y compris ses actes violents, qu'il s'agisse des attaques menées contre les groupes d'opposition ou des actions de l'État. Aux côtés d'autres événements de l'histoire de la Russie, la victoire de 1945 fournit la principale ressource symbolique destinée à justifier ou réfuter les revendications territoriales, l'ingérence dans les conflits intérieurs des pays étrangers, etc. Les énoncés historiques utilisés, descriptifs, optatifs ou performatifs, leur servent à justifier l'agression ou la riposte contre un ennemi extérieur - à l'instar des uniformes militaires de la Seconde Guerre mondiale utilisés par les militants du NOD pour légitimer leur attaque contre l'association Memorial. En abandonnant leurs mots d'ordre de transformation de la vie politique, les courants d'opposition doivent consolider leur discours autour de la défense de "positions morales " et dénoncer les actions du pouvoir par l'intermédiaire d'analogies historiques. Les invocations de la figure de l'opposant assassiné Boris Nemtsov sont ainsi devenues leur principale ressource symbolique. Le recours au passé dans les manifestations de rue ne vise donc pas tant à revendiquer ou à légitimer des transformations politiques - même s'il joue aussi ce rôle -, il est d'abord un instrument pour affirmer son identité politique, sa " ligne de front ", comme disent parfois les citoyens politiquement actifs en Russie ; ou, comme l'écrivait Martin Luther, ce qui fait qu'on se dresse car on ne peut rien faire d'autre (" hier stehe ich und ich kann nicht anders"). 


\section{Tableau 1. Types d'énoncés historiques relevés dans les manifestations de rue entre 2011 et 2016}

\begin{tabular}{|c|c|c|}
\hline Dénomination et description & $\begin{array}{l}\text { Proportion des énoncés } \\
\text { correspondant à ce profil parmi } \\
\text { tous les énoncés inventoriés } \\
\text { comportant une référence } \\
\text { historique }\end{array}$ & Exemples \\
\hline $\begin{array}{l}\text { Descriptifs : comparaison d'un } \\
\text { événement contemporain avec } \\
\text { un événement historique }\end{array}$ & $71,6 \%$ & $\begin{array}{l}\text { "La Syrie, nouvel Afghanistan?" } \\
\text { "1917-20 ?? " (légende d'un } \\
\text { portrait de Poutine dont l'ombre } \\
\text { du profil dessine celui de Staline) } \\
\text { " Bientôt un siècle que le pouvoir } \\
\text { en place fait souffrir la Russie» }\end{array}$ \\
\hline $\begin{array}{l}\text { Optatifs : expression d'un } \\
\text { souhait ou d'une volonté de } \\
\text { changer le présent par analogie } \\
\text { avec un fait historique }\end{array}$ & $12,6 \%$ & $\begin{array}{l}\text { « } 5 \text { victoires légendaires de l'armée } \\
\text { russe contre la Turquie : } \\
\text { - } 1770 \text { : bataille de Tchesmé, } \\
\text { amiral Ouchakov. } \\
\text { - } 1787 \text { : prise d'Izmaill, Souvorov } \\
{[\ldots]} \\
2015 \text { : ?» }\end{array}$ \\
\hline $\begin{array}{l}\text { Vocatifs : invocation } \\
\text { d'une figure historique }\end{array}$ & $13,3 \%$ & $\begin{array}{l}\text { «Andreï Dmitrievitch [Sakharov] } \\
\text { avec nous!" }\end{array}$ \\
\hline $\begin{array}{l}\text { Performatifs : énoncés } \\
\text { exprimant la valeur émotive } \\
\text { accordée par le destinateur } \\
\text { à une référence historique }\end{array}$ & $1,5 \%$ & $\begin{array}{l}\text { "Ces dates me font honte : 1939, } \\
\text { 1968, } 2014 \text { " } \\
\text { "Je suis fier de la victoire dans la } \\
\text { guerre de 1941-1945" }\end{array}$ \\
\hline $\begin{array}{l}\text { Réflexifs : réflexion sur } \\
\text { l'utilisation de l'histoire } \\
\text { à des fins politiques }\end{array}$ & $0,9 \%$ & $\begin{array}{l}\text { "Nous n'avons pas besoin d'une } \\
\text { histoire alternative " } \\
\text { "Je veux apprendre l'histoire, } \\
\text { pas de la propagande" }\end{array}$ \\
\hline
\end{tabular}

Tableau 2. Part des références historiques dans les slogans des manifestations de rue (en \% de l'ensemble des énoncés forts recensés)

\begin{tabular}{|l|c|c|c|c|}
\hline Types de manifestations & $\mathbf{2 0 1 1}$ & $\mathbf{2 0 1 4}$ & $\mathbf{2 0 1 5}$ & $\mathbf{2 0 1 6}$ \\
\hline $\begin{array}{l}\text { Manifestations } \\
\text { de l'opposition }\end{array}$ & $5 \%$ & $12 \%$ & $8 \%$ & $13 \%$ \\
\hline Manifestations loyalistes & Pas de données & Pas de données & $10 \%$ & $29 \%$ \\
\hline $\begin{array}{l}\text { Manifestations } \\
\text { de revendication sociale }\end{array}$ & Pas de données & $3 \%$ & $6 \%$ & $3 \%$ \\
\hline $\begin{array}{l}\text { Manifestations partisanes } \\
\text { de forces politiques } \\
\text { de gauche ou de droite }\end{array}$ & Pas de données & $7 \%$ & $6 \%$ & $3 \%$ \\
\hline
\end{tabular}


Tableau 3. Typologie des énoncés historiques utilisés dans les slogans des différentes manifestations (en \% de l'ensemble des slogans recensés comportant une référence historique)

\begin{tabular}{|l|c|c|c|c|c|}
\hline $\begin{array}{l}\text { Typologie des } \\
\text { énoncés / } \\
\text { Typologie des } \\
\text { manifestations }\end{array}$ & Descriptifs & Optatifs & Vocatifs & Performatifs & Réflexifs \\
\hline $\begin{array}{l}\text { Manifestations } \\
\text { de l'opposition }\end{array}$ & $31 \%$ & $19 \%$ & $47 \%$ & $2 \%$ & $2 \%$ \\
\hline $\begin{array}{l}\text { Manifestations } \\
\text { loyalistes }\end{array}$ & $61 \%$ & $25 \%$ & $7 \%$ & $2 \% \%$ \\
\hline $\begin{array}{l}\text { Manifestations } \\
\text { de } \\
\text { revendication } \\
\text { sociale }\end{array}$ & $26 \%$ & $51 \%$ & $17 \%$ & $2 \% \%$ \\
\hline $\begin{array}{l}\text { Manifestations } \\
\text { partisanes }\end{array}$ & $32 \%$ & $14 \%$ & $55 \%$ & $2 \%$ & $0 \%$ \\
\hline
\end{tabular}

Tableau 4. Évolution des énoncés historiques utilisés dans les meetings d'opposition (en \% du total des énoncés historiques utilisés dans les meetings d'opposition pour une année donnée)

\begin{tabular}{|l|c|c|c|c|}
\hline $\begin{array}{l}\text { Types d'énoncés } \\
\text { historiques }\end{array}$ & $\mathbf{2 0 1 1}$ & $\mathbf{2 0 1 4}$ & $\mathbf{2 0 1 5}$ & $\mathbf{2 0 1 6}$ \\
\hline Descriptifs & $12 \%$ & $48 \%$ & $41 \%$ & $17 \%$ \\
\hline Optatifs & $50 \%$ & $27 \%$ & $15 \%$ & $9 \%$ \\
\hline Vocatifs & $31 \%$ & $19 \%$ & $39 \%$ & $73 \%$ \\
\hline Performatifs & $0 \%$ & $4 \%$ & $1 \%$ & $1 \%$ \\
\hline Réflexifs & $4 \%$ & $2 \%$ & $3 \%$ & $0 \%$ \\
\hline
\end{tabular}

